\title{
Fluid management knowledge in hospital physicians: 'Greenshoots' of improvement but still a cause for concern
}

\author{
Authors: Richard Leach, ${ }^{\mathrm{A}}$ Siobhan Crichton, ${ }^{\mathrm{B}}$ Neil Morton, ${ }^{\mathrm{C}}$ Marc Leach ${ }^{\mathrm{D}}$ and Marlies Ostermann ${ }^{\mathrm{E}}$
}

\begin{abstract}
Fluid management is an essential competency for hospital doctors, but previous studies suggest junior clinicians lack the necessary 'knowledge' and 'prescription skills' to complete this task, resulting in preventable morbidity and mortality. In this study, preregistration $(n=146)$, core $(n=66)$ and specialty $(n=133)$ medical trainees and general medical consultants ( $n=11$ ) completed a structured questionnaire exploring fluid management training, confidence, serious adverse event experience and a 20 -item fluid management 'knowledge' test. Results were compared with those of intensive care consultants $(n=20)$. Most clinicians reported limited training and extensive 'unreported' serious adverse events experience. Knowledge about fluid and electrolyte requirements, fluid composition and chloride toxicity had improved compared to historical reports but overall test scores (median (interquartile range (IQR)): with a maximum score of 20 ) were low. Foundation year trainees scored 7 (IQR 5-8), core medical trainees scored 9 (IQR 6-10), specialist registrars scored 8 (IQR 6-10) and general medical consultants scored 8 (IQR 6-12) compared with the intensive care consultant score of 16 (IQR 14-16). Although weakly correlated, fluid management 'confidence' appeared higher than 'knowledge' tests would justify. These results suggest that physicians' fluid management knowledge is inadequate, including that of senior colleagues, compounded by poor training and failure to learn from serious adverse events.
\end{abstract}

KEYWORDS: Fluid management, serious adverse events, education, training

DOI: $10.7861 /$ clinmed.2019-0433

\section{Introduction}

The assessment, prescription and administration of fluids and electrolytes are important daily tasks on hospital wards but

Authors: ${ }^{\text {A }}$ linical director of pulmonary and critical care medicine, Guy's and St Thomas' NHS Foundation Trust, London, UK;

${ }^{B}$ statistician, University College London, London, UK; ${ }^{C}$ junior clinical research fellow, Guy's and St Thomas' NHS Foundation Trust,

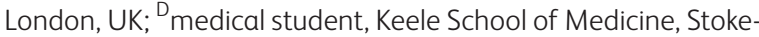
on-Trent, UK; ${ }^{E}$ clinical lead in critical care medicine, Guy's and St Thomas' NHS Foundation Trust, London, UK junior clinicians, who are responsible for most prescriptions of intravenous (IV) fluid and electrolytes, lack the 'core' knowledge and practical assessment skills to undertake this task. ${ }^{1-5}$ Many are unaware of these knowledge deficits and are inappropriately confident in their prescribing skills. ${ }^{6}$ Failure to report and learn from serious adverse events (SAEs) further compounds the situation. Less is known about senior clinicians' fluid management knowledge and confidence and, although it is assumed to be adequate, many received little formal teaching or assessment during their training.

The National Institute for Health and Care Excellence (NICE) published its clinical guideline Intravenous fluid therapy in adults in hospital: Clinical guideline [CG174] in 2013. ${ }^{2}$ Recent publications describe methods to improve teaching, prescribing and guideline compliance, but these reports also suggest that clinicians' inadequate knowledge remains a significant issue..$^{7-10}$ We aimed to assess ongoing acquisition and current knowledge of 'medical' hospital clinicians, including middle and senior grade practitioners. We explored previous training, experience of and learning from SAEs, confidence with IV fluid prescription and current knowledge based on NICE CG174. Performance was compared with that of intensive care consultants (ICC), who represented the best available clinical standard for comparison (in view of their specialist fluid management training), and historical knowledge assessments from 2001-2016., ${ }^{1,6,11,12}$

\section{Methods}

Permission to collect questionnaire data was obtained before the study from training programme directors. Data collection occurred between January 2016 and December 2018. Foundation year doctors (FYs), core medical trainees (CMTs) and specialist registrars ( $\mathrm{SpRs}$ ) completed a supervised, anonymous, structured questionnaire in the 35-40 minutes before a fluid management teaching session within their training programme. Answers to the 'knowledge test' multiple choice questions (MCQ) were provided during teaching. A few FYs and SpRs repeated the MCQs immediately after teaching (matched with the initial test) or 6-12 months later on subsequent training days (not matched with previous anonymised questionnaires).

Data for FY and CMT ('junior' clinicians) was collected at $>10$ formal, supervised lunchtime training sessions in two south London teaching hospitals. SpRs ('middle grade' clinicians) completed questionnaires during two formal general internal medicine (GIM) training days in north and south London and two pan-London 
specialty training days in acute and respiratory medicine. SpR participants were all in London training programmes and worked in associated Greater London hospitals. Additional sessions were arranged for intensive care unit (ICU) trainees who were unable to attend formal teaching opportunities. Consultants ('senior' clinicians) were individually invited to participate and complete the questionnaire without recourse to additional information. Thirtyfive questionnaires were distributed to both ICCs and general medical consultants (GMCs) at two teaching and one district general hospital. Questionnaire completion by GMCs and ICCs was not supervised in all but six (two ICCs and four GMCs).

\section{Questionnaire}

The questionnaire (see supplementary material S1) was designed by the authors, and modified in response to advice from educational colleagues, previous questionnaire authors, NICE guideline colleagues and following trials before the survey. The 'degree of difficulty' of the 'knowledge' MCQs was graded to represent a meaningful assessment across grades of increasing seniority. The questionnaire collected information in five categories.

> Background data: medical grade and specialty (eg respiratory)

$>$ Assessment of previous training: hours of fluid management training completed as undergraduates and postgraduates using a scale: $<5$ hours, $5-10$ hours, $10-20$ hours, $20-40$ hours and $>40$ hours.

> Experience and management of fluid-related SAEs: yes/ no, and if 'yes' how often, using a scale: $<5,5-10,10-20$ and $>20$. Participants were asked to indicate if these events were reported using a scale: always, usually, occasionally, rarely and never. Respondents were also asked if their fluid management had resulted in SAEs and if 'yes' whether they were reported (yes, no or rather not say).

> Confidence in IV fluid management: respondents rated their confidence on a 10-point scale $(0=$ no confidence to $10=$ very confident) before and after completing the 'knowledge' MCQs.

$>$ 'Knowledge' test: this assessed key NICE guidance priorities and compared performance with previous fluid management knowledge assessments between 2001-2016. 1,6,11,12 Twenty 'single best answer of five' MCQs were utilised. Five examined 'essential' knowledge and had been used in previous questionnaires (eg daily water/electrolyte requirements, sodium content of $0.9 \%$ saline and commonly used fluid compositions). A further five questions measured 'desirable' knowledge (eg normal chloride level, body water content and saline-induced hyperchloraemic metabolic acidosis) also used in previous questionnaires. Four questions were clinical 'scenarios' addressing NICE CG174 assessment, resuscitation, maintenance and replacement algorithms. Six questions were aimed at experienced physicians and addressed renal function (eg daily solute excretion), intravascular volume maintenance and electrolyte redistribution during illness.

\section{Statistical analysis}

Responses were summarised as frequency (percentage) and compared across grades using the $\chi^{2}$ or Fisher's exact test, as appropriate. Two scores were created for the five questions relating to 'essential' and 'desirable' knowledge reflecting the number of items answered correctly. A further score was calculated for all 20 questions. Scores were summarised as median (interquartile range (IQR)) and compared across grades using the KruskalWallis test. Confidence scores before and after completing the questionnaire were summarised as median (IQR) and compared using the Wilcoxon signed-rank test and Spearman's rank correlation coefficient $\left(r_{s}\right)$ to assess the relationship between confidence and knowledge. Changes in knowledge scores of participants who repeated the questionnaire after the training session were also compared using the Wilcoxon signed-rank test. Analysis was conducted using Stata 15/IC (StataCorp, College Station, USA).

\section{Results}

\section{Participants}

Three-hundred and seventy-six respondents completed the questionnaire. Twenty were ICCs (11 anaesthetic, four medicine and five intensive care medicine trained), 11 were GMCs (four acute, four respiratory, one renal, one diabetes/endocrinology and one elderly care), 133 were SpRs (56 respiratory, 18 gastroenterology, 14 ICU, 13 elderly care, 12 diabetes/ endocrinology, 10 rheumatology, five acute medicine, two renal and three others), 66 were CMTs and 146 were FYs.

\section{Fluid management training}

Table 1 shows the number of hours of undergraduate and postgraduate fluid management training. Overall, $69 \%$ of respondents reported $<10$ hours teaching during undergraduate training and $75 \%<10$ hours during postgraduate training. Junior and middle grade doctors reported more undergraduate teaching than senior doctors (ICCs and GMCs) but senior doctors more postgraduate training than junior colleagues.

\section{Fluid management confidence}

Confidence varied by grade and was highest in ICCs (median 9 (IQR 8-9)) and lowest in FYs (5 (IQR 4-7)). Overall 373 respondents reported a mean confidence score of 7 (IQR 5-7) before completing the questionnaire. Confidence fell by a median of 2 (IQR 1-3) points to 4 (IQR 2-5) after completing the questionnaire $(n=292 ; p<0.001)$. Only $50(17 \%)$ respondents felt as confident (or better) after the questionnaire. Table 2 summarises the correlation $\left(r_{s}\right)$ between confidence and 'knowledge test' scores before and after test performance. Confidence was significantly, but weakly, correlated with performance before and, more so, after the test when all participants were analysed. The correlation was weaker when ICCS were excluded.

\section{Experience of SAEs}

Overall, $70 \%$ of respondents had SAE management experience, which varied by grade $(100 \%$ of ICCs/GMCs, $>90 \%$ of SpRs/ CMTs and $32 \%$ of FYs; see supplementary material S2). Reporting was sporadic with $>70 \%$ indicating SAEs were 'never' or 'rarely' reported and $5 \%$ that they were 'always' or 'usually' reported. Fifty per cent of ICCs, $45 \%$ of GMCs, $28 \%$ of SpRs, $14 \%$ of CMTs and $1 \%$ of $\mathrm{FYs}$ stated that their fluid management had resulted in SAEs. Only $16 \%$ of these were reported and seniority did not 
Table 1. Number of hours of undergraduate and postgraduate training reported by study participants

\begin{tabular}{|c|c|c|c|c|}
\hline & All & $\begin{array}{l}\text { ICC and } \\
\text { GMC }\end{array}$ & SpR & $\begin{array}{l}\mathrm{CMT} \\
\text { and } \mathrm{FY}\end{array}$ \\
\hline $\begin{array}{l}\text { Total } \\
\text { participants, n }\end{array}$ & 376 & 31 & 133 & 212 \\
\hline $\begin{array}{l}\text { Undergraduate } \\
\text { training } \\
\text { responses, } \mathrm{n}\end{array}$ & 359 & 31 & 124 & 205 \\
\hline$<5$ hours, $n(\%)$ & $171(47)$ & $22(71)$ & $42(34)$ & $107(52)$ \\
\hline 5-10 hours, $n(\%)$ & $79(22)$ & $1(3)$ & $29(23)$ & $49(24)$ \\
\hline 10-20 hours, $n(\%)$ & $37(11)$ & 0 & $12(10)$ & $25(12)$ \\
\hline $20-40$ hours, $n(\%)$ & $21(6)$ & $2(6)$ & $10(8)$ & $9(4)$ \\
\hline$>40$ hours, $n(\%)$ & $51(14)$ & $5(16)$ & $31(25)$ & $15(7)$ \\
\hline $\begin{array}{l}\text { Postgraduate } \\
\text { training } \\
\text { responses, } n\end{array}$ & 337 & 30 & 123 & 184 \\
\hline$<5$ hours, $n(\%)$ & $193(57)$ & $5(17)$ & $37(30)$ & $151(82)$ \\
\hline 5-10 hours, $n(\%)$ & $60(18)$ & $9(30)$ & $33(27)$ & $18(10)$ \\
\hline $10-20$ hours, $n(\%)$ & $25(8)$ & $3(10)$ & $17(14)$ & $5(3)$ \\
\hline 20-40 hours, n (\%) & $21(6)$ & $9(30)$ & $8(7)$ & $4(2)$ \\
\hline$>40$ hours, $n(\%)$ & $38(11)$ & $4(13)$ & $28(22)$ & $6(3)$ \\
\hline
\end{tabular}

$\mathrm{CMT}=$ core medical trainee; $\mathrm{FY}=$ foundation year doctor; $\mathrm{GMC}=$ general medical consultant; ICC = intensive care consultant; $\mathrm{SpR}=$ specialist registrar.

increase the likelihood of 'self-confessed' SAEs being reported $(p=0.814)$.

\section{Knowledge test}

Supplementary material S3 Table S1 shows the percentage of senior, middle grade and junior doctors correctly answering the essential knowledge questions and the grouped scores for 'essential' (Score 1; maximum score 5), 'desirable' (Score 2; maximum score 5) and 'overall' test scores (Score 3; maximum score 20). In the five 'essential' knowledge questions, GMCs, middle grade and junior doctor scores were significantly lower than ICCs $(p<0001)$. ICCs answered a median of 5 (IQR 4-5) questions correctly compared to a median of 2 (IQR 2-3) for 'medical' practitioners (ie GMCs, SpRs, CMTs and FYs) which did not vary with seniority $(p=0.459)$. Overall, $50 \%$ or less of 'medical' practitioners knew normal daily water/electrolyte requirements and commonly used fluid compositions, although two-thirds correctly identified the sodium chloride content of $0.9 \%$ saline (supplementary material S3 Table S2). For the five 'desirable' knowledge questions, ICCs answered a median of 4 (IQR 3-5) questions correctly compared with a median of 2 (IQR 1-3) for medical practitioners.

Supplementary material S3 Table S3 shows the percentage of senior, middle grade and junior doctors correctly answering individual 'desirable' knowledge and NICE scenario-based questions. For all 20 MCQs, ICCs scored highest $(p<0.001)$ answering 16 (IQR 13-16) correctly compared with GMCs answering 8 (IQR 6-12), SpRs answering 8 (IQR 6-10), CMTs answering 9 (IQR 6-10) and FYs answering 7 (IQR 5-7). For SpRs, the 'overall' test scores (maximum score 20) differed significantly across specialties $(p=0.002)$ with ICU SpRs scoring 11 (IQR 9-12), diabetes/endocrinology scoring 9 (IQR 7-11), respiratory scoring 8 (IQR 6-9), gastroenterology scoring 7 (IQR 5-9), elderly care scoring 7 (IQR 6-8) and other specialties scoring 8 (IQR 7-10). However, SpR 'essential' knowledge scores did not differ $(p=0.3$ ) with a median of 2 (IQR 2-3) across all specialities.

\section{Repeat knowledge tests after training}

Forty respondents (23 SpRs, three CMTs and $14 \mathrm{FYs}$ ) completed a second questionnaire after training on fluid management; 32 (18 SpRs and $14 \mathrm{FYs}$ ) before and after questionnaires could be linked. Knowledge improved significantly $(p<0.001$; supplementary material S3 Table S3) and respondents correctly answered an additional 2 (IQR 1-2) essential, 1 (IQR 2-5) desirable and 6 (IQR 4.5-8) other MCQs.

\section{Discussion}

This study suggests that the fluid management 'knowledge base' is poor across the whole 'medical' workforce including middle grade and senior physicians. Essential, desirable and overall knowledge scores were significantly lower for all grades compared to ICCs (the best available comparator group). Despite poor overall scores, with a median of only two of five 'essential' and two of five 'desirable' knowledge questions answered correctly, there were 'greenshoots' of improvement compared to previous assessments. In postoperative patients, poor fluid and electrolyte management prolongs hospital stay and increases cost, morbidity and mortality. ${ }^{3-5,13-16}$ Between $17-54 \%$ of postoperative cases experience at least one fluid related complication due to under/over hydration and/or harmful electrolyte imbalance (eg tachyarrhythmia). ${ }^{3,14,15}$ The poor fluid management knowledge of junior clinicians is well documented and has remained a concern in recent studies. ${ }^{6,15-19}$ Surgical SpRs and consultants perform slightly better than their juniors but little is known about the knowledge of 'senior medical' clinicians. ${ }^{20}$ In postoperative surgical patients, junior clinicians are responsible for $>85 \%$ of fluid prescriptions. ${ }^{1,3,20}$ However, in medical practice, middle grade clinicians (eg SpRs) often 'direct' fluid management, which is subsequently 'written up' by junior colleagues. In an 'apprenticeship-style' training system, like GIM, inadequate senior clinician 'knowledge' is likely to impact junior colleagues training.

Our study is concerning but provides some optimism, too. FY and CMT fluid management 'knowledge' could be compared with results from four previous studies between 2001-2016. 1,6,11,12 $^{2}$ Identical or very similar questions were used (and the data recorded) in these previous reports and our study. For example, $65 \% \mathrm{FY}$ and $73 \% \mathrm{CMT}$ correctly identified the sodium chloride content of $0.9 \%$ saline in our study compared to $21-37 \%$ and $46-51 \%$, respectively, in previous reports. Reassuringly, this question was also correctly answered by $>75 \%$ of SpRs and consultants. In addition, $\sim 50 \%$ of $\mathrm{FYs}$ in this study knew the recommended daily water and sodium requirements compared to $6-33 \%$ in previous reports. Also, $34 \%$ of FYs correctly identified the composition of Hartmann's solution compared to $8-13 \%$ previously, although less 
Table 2. Correlation $\left(r_{s}\right)$ between confidence and knowledge test scores (see score measures) before and after test performance

\section{Score 1 essential knowledge,} $r_{s}$ ( $p$ value)

All

Confidence before

$0.20(<0.001)$

$0.28(<0.001)$

Confidence after

All - excluding ICCs

Confidence before

$0.10(0.052)$

Confidence after

$0.17(0.05)$

ICCs

Confidence before

$0.23(0.324)$

$0.22(0.434)$

Confidence after

\section{GMCs}

Confidence before

$-0.01(0.988)$

$0.63(0.072)$

Confidence after

\section{SpRs}

Confidence before

Confidence after

\section{CMTs}

Confidence before

Confidence after

FYs

Confidence before

Confidence after
$0.22(0.008)$

$0.07(0.400)$

$-0.02(0.862)$

$-0.18(0.158)$

$-0.01(0.928)$

$0.28(0.002)$

\section{Score 2 desirable knowledge,} $r_{s}$ ( $p$ value)

Score 3 all,

$r_{s}$ ( $p$ value)

$0.22(<0.001)$

$0.21(<0.001)$

$0.30(<0.001)$

$0.31(<0.001)$

$0.14(0.008)$

$0.11(0.037)$

$0.21(<0.001)$

$0.21(<0.001)$

$0.37(0.107)$

$0.27(0.242)$

$0.30(0.274)$

$0.41(0.129)$

$0.22(0.517)$

$0.01(0.970)$

$0.77(0.015)$

$0.49(0.183)$

$0.03(0.773)$

$0.00(0.914)$

$-0.24(0.045)$

$-0.18(0.128)$

$0.11(0.400)$

$-0.079(0.527)$

$0.18(0.151)$

$0.25(0.044)$

$0.02(0.827)$

$0.05(0.543)$

$0.11(0.207)$

$0.18(0.044)$

$\mathrm{CMT}=$ core medical trainee; $\mathrm{FY}=$ foundation year doctor; GMC = general medical consultant; $\mathrm{ICC}=$ intensive care consultant; SpR = specialist registrar.

improvement was seen for Gelofusine composition ( $9 \%$; previously $4 \%$ ), probably related to recent reductions in the use of colloid solutions. Earlier this year, a similar study in FYs also reported an improvement in the knowledge of daily water/electrolyte requirements but not of fluid composition (only $31 \%$ knew the sodium content of $0.9 \%$ saline). $^{20}$ 'Desirable' knowledge also improved compared to historical assessments, with most clinicians aware of the metabolic risks of excess saline. However, less than $50 \%$ knew the normal serum chloride or intracellular electrolyte concentrations. Overall ICU SpRs scored better than GMCs and medical SpRs, possibly due to training factors or greater ICC knowledge in an 'apprenticeship teaching system'. Nevertheless, despite the improvements reported above, $50 \%$ or more of medical practitioners still did not know the daily fluid and electrolyte requirements recommended in NICE CG174 or the electrolyte compositions of commonly used fluids (supplementary material S3).

In four questions using NICE CG174 recommendations to test 'typical clinical fluid management scenarios', the best intravenous fluid and electrolyte 'maintenance' regimen for an elderly comorbid patient unable to drink was correctly identified by $75 \%$ of ICCs but, disappointingly, only $17-30 \%$ of 'medical' practitioners. However, two questions addressing the best 'replacement' regimen in a complex postoperative patient with large nasogastric losses and the recommended use of a physiologically balanced fluid for a high-volume resuscitation were correctly answered by most senior/middle grade medical practitioners (but $<50 \%$ of $\mathrm{FYs}$ ). In the final complex question, $\leq 50 \%$ of respondents recognised that the oliguria in a recovering trauma case was due to reduced intravascular volume.

Junior clinicians' fluid management confidence has previously been reported to be greater than 'knowledge test' results suggest is reasonable. ${ }^{1,6,12}$ In this study, pre-test confidence was weakly but significantly correlated with test results across all participants (Table 2). This correlation was stronger in ICCs (but not significant). When ICC participants were excluded from the analysis, the correlation in remaining participants was weaker, but still significant, consistent with some pre-test recognition of knowledge 'weakness'. In terms of patient safety, lack of awareness of knowledge deficits, described as a failure of metacognition, is a concern. Metacognition describes the awareness of one's own knowledge and the ability to reflect on, evaluate and address deficits in self-knowledge. ${ }^{21,22}$ Although less of an issue in the specialty aspects of a physician's practice, it probably does impact on 'general' patient care (eg nutrition or falls management). ${ }^{21}$ Failure of metacognition is as likely to occur in senior clinicians as it is in junior clinicians. 
SAEs reviews effectively address 'knowledge deficits', improves practice, prevents recurrence and enhances team learning. This study demonstrates a failure of this 'safety' mechanism in fluid management. Although most practitioners frequently manage fluid-related SAEs, and $\sim 50 \%$ of senior clinicians have caused a SAE, few were reported or investigated. Consequently, this vital aspect of learning is lost. Improving SAE review presents an opportunity to promote learning and partly addresses the failure of the 'apprenticeship system'. The 'test, teach and retest' strategy was also an effective training tool as demonstrated in the 40 participants who redid the test, answering an additional nine questions correctly.

There were study limitations. Although trainee and ICC numbers were robust, it was difficult to 'persuade' GMCs to participate. Only 11 GMCs completed the questionnaire (31\%). This limited consultant participation and variability in group sizes is a weakness. In addition, few senior colleagues ( $20 \%)$ were prepared to complete the questionnaire supervised. Other issues included the reduction in 'colloid fluid' use during the study, rendering an 'essential' knowledge question about Gelofusine less relevant and, for reasons of anonymity, post teaching scores could not be matched. Finally, data collection occurred over a 3-year period, potentially allowing increased 'penetrance' of NICE guideline knowledge into clinical practice. Although this appeared to be a factor, we were unable to demonstrate a significant effect.

This study suggests that fluid management knowledge remains inadequate across the hospital medical workforce, including senior colleagues. However, improvement has occurred in specific areas (eg daily water/electrolyte requirements) compared to historical reports. The weak correlation between confidence and 'test' scores suggests physicians do have awareness of this issue, but in these circumstances traditional 'apprenticeship systems' of learning are unlikely to be effective, compounded by poor training and failure to learn from SAEs. We propose that undergraduate and postgraduate training should be standardised and based on NICE CG174. It should be delivered by those with appropriate knowledge and adequately assessed within training programmes. Senior clinicians need to be made aware of this potential deficiency in their knowledge base and appropriate training material made available with greater emphasis on this topic in 'refresher' courses and 'specialist' conferences.

\section{Key points}

> Fluid management knowledge remains inadequate across the hospital medical workforce including senior clinicians.

> Doctors' 'confidence' in fluid management appears higher than 'knowledge' tests justify.

$>$ Specific aspects of fluid management knowledge have improved compared to historical reports.

> Failure to report and learn from serious adverse events is a major problem in fluid management.

$>$ Improvement in fluid management training is required with less emphasis on 'apprenticeship-style' teaching.

$>$ Better learning from serious adverse event reviews is an opportunity to promote improvement.

\section{Supplementary material}

Additional supplementary material may be found in the online version of this article at www.rcpjournals.org/clinmedicine:
S1 - Fluid management questionnaire.

S2 - Table showing clinician experience with serious adverse events related to fluid management.

S3 - Tables showing knowledge questionnaire test scores: 'Essential' knowledge scores and 'overall' scores, and 'desirable' knowledge and 'NICE' scenario questions.

\section{Acknowledgements}

We would like to acknowledge the help of the postgraduate education teams across both north and south London for their help with this study.

\section{References}

1 Lobo DN, Dube MG, Neal KR et al. Problems with solutions: drowning in the brine of an inadequate knowledge base. Clin Nutr 2001;20:125-30.

2 Leach RM. Fluid management in hospital patients. Clin Med 2010:10:611-5.

3 National Institute for Health and Care Excellence. Intravenous fluid therapy in adults in hospital: Clinical guideline [CG174]. NICE, 2013. www.nice.org.uk/guidance/cg174/resources/intravenousfluid-therapy-in-over-16s-in-hospital-35109752233669

4 Powell-Tuck J, Gosling P, Lobo DN et al. Summary of the British Consensus Guidelines on Intravenous Fluid Therapy for Adult Surgical Patients (GIFTASUP) - For Comment. J Intensive Care Soc 2009;10:13-5

5 Callum KG, Gray AJG, Hoile RW et al. Extremes of age: The 1999 report of the National Confidential Enquiry into Perioperative Deaths. London: National Confidential Enquiry into Perioperative Deaths, 1999. www.ncepod.org.uk/1999report/99full.pdf

6 Scott N, Squibbs J, Lake A, Guha A. Risks of intravenous fluid therapy by first year residents - a prospective study. $\mathrm{Br}$ ] Med Med Res 2016:15:1-13.

7 Sansom LT, Duggleby L. Intravenous fluid prescribing: Improving prescribing practices and documentation in line with NICE CG174 guidance. BMJ Qual Improv Rep 2014;3:u205899.w2409.

8 Walker GE, Stewart-Parker E, Chinthapalli S et al. Intravenous fluid use in the acutely unwell adult medical inpatient: improving practice through a clinical audit process. J $R$ Coll Physicians Edinb 2012:42:211-5.

9 Awad S, Allison SP, Lobo DN. Fluid and electrolyte balance: The impact of goal directed teaching. Clin Nutr 2008;27:473-8.

10 Gnanasampanthan V, Porten L, Bissett I. Improving surgical intravenous fluid management: a controlled educational study. AZN J Surg 2014:84:932-6.

11 Thevelin S, Miller G, Guthrie E, Portlock JC. CP-174 Healthcare professionals' knowledge of intravenous fluid treatment. Eur ] Hosp Pharm 2015;22(Suppl 1):A72-2.

12 Powell AGMT, Walker VC, Paterson-Brown L, Clark G, Drummond $\mathrm{GB}$, Paterson-Brown S. Intravenous fluid prescribing knowledge and confidence in F1 doctors. Ann R Coll Surg Engl 2013;95:1-6.

13 De Silva AN, Scibelli T, Itobi E et al. Symposium 3: Death by drowning. Improving peri-operative fluid management in a large teaching hospital: pragmatic studies on the effect of changing practice. Proc Nutr Soc 2010;69:499-507.

14 Walsh SR, Walsh C]. Intravenous fluid associated morbidity in postoperative patients. Ann R Coll Surg Engl 2005;87:126-30.

15 Walsh SR, Cook EJ, Bentley R et al. Peri-operative fluid management: prospective audit. Int J Clin Pract 2008;62:492-7.

16 Arieff AI. Fatal postoperative pulmonary edema: pathogenesis and literature review. Chest 1999:115:1371-7.

17 Lim CT, Dunlop M, Lim CS. Intravenous fluid prescribing practices by foundation year one doctors - a questionnaire study. JRSM Short Rep 2012:3:64-6. 
18 Lobo DN, Dube MG, Neal KR, Allison SP, Rolands B]. Peri-operative fluid and electrolyte management: a survey of consultant surgeons in the UK. Ann R Coll Surg Engl 2002:84;156-60.

19 Lupi MM, Maweni RM, Shirazi S, Wali UJ. Fluid and electrolyte balance-establishing the knowledge base of Foundation Year One doctors. Ir J Med Sci 2019;188:1047-55.

$20 \mathrm{Pal}$ A, Conveney E. Intravenous fluids: do doctors know what they are prescribing. Ann R Coll Surg Engl (suppl) 2012;94:211-3.

21 Gonullu I, Artar M. Metacognition in medical education. Educ Health 2014;27:225-6.
22 Colbert C, Graham L, West C et al. Teaching metacognitive skills: Helping your physician trainees in the quest to 'know what they don't know'. Am J Med 2015;128:318-24.

Address for correspondence: Dr Richard Leach, Departments of Medicine and Critical Care Medicine, St Thomas' Hospital, Westminster Bridge Road, London SE1 7EH, UK.

Email: richard.leach@gstt.nhs.uk 